Cite as: Nemeth, D., Hallgató, E., Janacsek, K., Sándor, T., \& Londe, Z. (2009). Perceptual and motor factors of implicit skill learning. Neuroreport, 20(18), 1654-1658.

\title{
Perceptual and motor factors of implicit skill learning
}

\author{
Dezso Nemeth $^{\text {a, }}{ }^{\text {** }}$, Emese Hallgató ${ }^{\mathrm{a}}$, Karolina Janacsek $^{\mathrm{a}}$, Timea Sándor ${ }^{\mathrm{a}}$, Zsuzsa \\ Londe $^{c}$
}

${ }^{a}$ Institute of Psychology, University of Szeged, Hungary

${ }^{\mathrm{b}}$ Center for the Brain Basis of Cognition, Georgetown University, Washington DC, USA

${ }^{c}$ American Language Institute, University of Southern California, Los Angeles, USA

* The first three authors contributed equally to this work

Running Title: Factors of implicit sequence learning

Corresponding author: Dezso Nemeth, Ph.D., Institute of Psychology, University of Szeged, Egyetem u. 2., 6722, Szeged, HUNGARY, Email: nemethd@edpsy.uszeged.hu, Tel.: +36 62544 692, Fax: +36 62544509

The authors report no conflict of interest and have no financial disclosure. 


\begin{abstract}
Implicit skill learning underlies not only motor but also cognitive and social skills, and represents an important aspect of life from infancy to old age. Earlier research examining this fundamental form of learning has demonstrated that learning relies on motor and perceptual skills, along with the possible role of oculomotor learning. The goals of the present study were to determine whether motor or perceptual cues provide better prompts to sequence learning and to remove the possibility of oculomotor learning during the task. We used a modified version of the probabilistic ASRT task, which allowed the separation of motor and perceptual factors. Our results demonstrated that motor and perceptual factors influenced skill learning to a similar extent.
\end{abstract}

Keywords: implicit skill learning; motor learning; perceptual learning; ASRT; oculomotor learning 


\section{INTRODUCTION}

Implicit skill learning occurs when information is acquired from an environment of complex stimuli without conscious access either to what was learned or to the fact that learning had occurred [1]. In everyday life, this learning mechanism is crucial for adapting to the environment and to evaluate events. The most important models of skill learning in cognitive neuroscience and neuropsychological studies emphasize the role of the basal ganglia and the cerebellum [2-4], while the role of the hippocampus remains inconclusive [5,6]. Skill learning can be differentiated into phases (an initial rapid phase and a subsequent slower phase), into types (motor, visuo-motor or perceptual such as visual, auditory, etc.), and into consciousness types (implicit and explicit) [2]. Implicit motor skill learning tasks have been used for decades, but there is no agreement about how these tasks reflect motor versus perceptual learning, and what their proportions are.

The most widely used task to measure skill learning is the SRT (Serial Reaction Time) task [7]. In this task, the stimulus appears in one of four possible positions on the screen and the subject has to press the appropriate response key as fast as possible. The stimuli follow a predefined sequence, and although the research subjects are not aware of this, they perform better on these trials than in corresponding random trials. In most SRT tasks, the location of the stimulus corresponds with the location of the response key. Therefore, learning can be influenced by the sequence of stimuli locations on the screen (perceptual learning), by the correct answer button sequence in the egocentric space (answer-based learning) or by the finger movement patterns (effector-based learning) [8].

Another disadvantage of these paradigms (classical SRT and finger-tapping tasks) is that after a short training session, the subjects often recognize the stimulus 
pattern, which causes significant limitations in studying implicit learning [9]. In contrast, using the Alternating Serial Reaction Time (ASRT) Task [9] allows researchers to overcome this aforementioned problem by employing an eight-element sequence, whereby random elements alternate with sequence elements (e.g.: 2-R-3-R-1$\mathrm{R}-4-\mathrm{R}$, where $\mathrm{R}$ refers to random).

In these research paradigms, it is difficult to isolate perceptual learning. Specifically, motor learning cannot be eliminated in both observation- and transferbased studies because it is the motor response reaction time that gives the informative measurements [10]. Perceptual learning in these paradigms can be observed only if it can be demonstrated in addition to implicit skill learning. For example, Robertson and Pascual-Leone [11] showed that if perceptual and motor sequences are combined (e.g., color and location) it leads to a greater level of learning than either one of the sequences alone.

In the case of first-order probability sequences, motor learning is not necessary to learn patterns. However, in second-order probability sequences (e.g., ASRT), perceptual learning is, at best, minimal [8]. Nevertheless, previous studies have been able to isolate perceptual learning based on second- or higher-order probability sequences [12]. For example, Dennis and colleagues [10] found that young adults showed implicit skill learning in higher-order sequences even without motor learning. Moreover, if no motor response was requested, deterministic sequence learning (e.g., SRT) led to explicit learning by simply observing the stimuli, whereby subjects revealed the hidden sequence explicitly $[9,13]$. In the case of second-order sequences, explicit knowledge has been shown to be minimal or totally eliminated [9]. Song et al. [14] demonstrated perceptual learning using similar task and found that learning took place 
even without a motor response to the observed stimuli. After the observation, subjects were able to transfer the sequence knowledge to the testing (motor) condition. The concern with this study was that the stimuli appeared on four different areas of the screen. Hence, skill learning could have reflected oculomotor learning as well [e.g., 14]. The question remains whether learning is purely perceptual when it is accompanied with eye movements. Remillard [8] found that perceptual learning was not influenced by the distance between the stimuli (i.e., the amplitude of the eye-movement). On the other hand, Willingham and colleagues [13] were not able to show perceptual learning without eye movements.

Willingham, Wells, Farrel and Stemwedel [15] changed the conditions of the SRT task after the learning phase in one of the two following ways: either the stimulus sequence (perceptual information) remained the same as in the learning phase while the sequence of the answers (motor information) was changed, or the motor response sequence remained the same and the response locations changed (subjects had to answer crossing their hands during the testing phase). Subjects were able to transfer their knowledge only if the sequence of response locations was maintained, not the sequence of finger movements [15]. These findings suggest that the sequence of response locations must have been retained in order for implicit knowledge to transfer, whereas the contribution of motor and perceptual information was less considerable. It is important to note that Willingham and colleagues [15] did not eliminate the possibility of oculomotor learning since the sequence occurred perceptually in the locations of the stimuli.

The goal of the present study was to investigate the role of perceptual learning in implicit sequence learning through a modified ASRT task. In this modified paradigm, 
the sequence followed a second-order regularity that eliminated the possibility of oculomotor learning because the stimuli always appeared in the same, central position. Similar to the Willlingham et al. [15] study in the learning phase, the sequence of stimuli and their responses were different. In the second phase (testing or transfer phase), the sequence of stimuli (perceptual information) remained the same and the response sequence (motor information) changed or vice versa.

Our hypothesis was that, unlike Willingham et al. [15], we would be able to show perceptual learning or perceptual transfer with a task that eliminated oculomotor learning. In addition, our goal was to create a task that would distinguish between perceptual and motor factors of implicit sequence learning.

\section{METHODS}

Participants - Thirty-four healthy right-handed subjects took part in the experiment. Half of the subjects were randomly assigned to the Perceptual condition (mean age $=21.76$ years, $\mathrm{SD}=2.02 ; 7$ male $/ 10$ female), and the other half were assigned to the Motor condition (mean age $=21.76$ years, $\mathrm{SD}=1.64 ; 8$ male/9 female). Subjects did not suffer from any developmental, psychiatric or neurological disorders. All subjects provided signed informed consent agreements and received no financial compensation for their participation.

Task - We used a modified version of the ASRT task [9], the so-called AS-RTRace. We created a story about a car race for the task. The stimuli were the left, right, up and down arrows $(5 \mathrm{~cm}$ long and $3 \mathrm{~cm}$ wide), which appeared in the center of the screen. When the stimulus appeared on the screen, it represented the car's direction. For example, when the subjects saw an up arrow, they had to press the up button on the 
keyboard to move the car forward, the left button to turn left, and so on. All subjects pressed the keys with their dominant hand.

After the starting block of 85 random presses, they were told that there was a car crash and the steering wheel failed (see Figure 1A). The car now kept going to the left if they wanted to go straight, but by turning the steering wheel right they could correct this malfunction, and could continue to go straight. Thus subjects had to mentally rotate the arrows (the steering wheel) by 90 degrees to the right, and press the button corresponding to this rotated arrow.

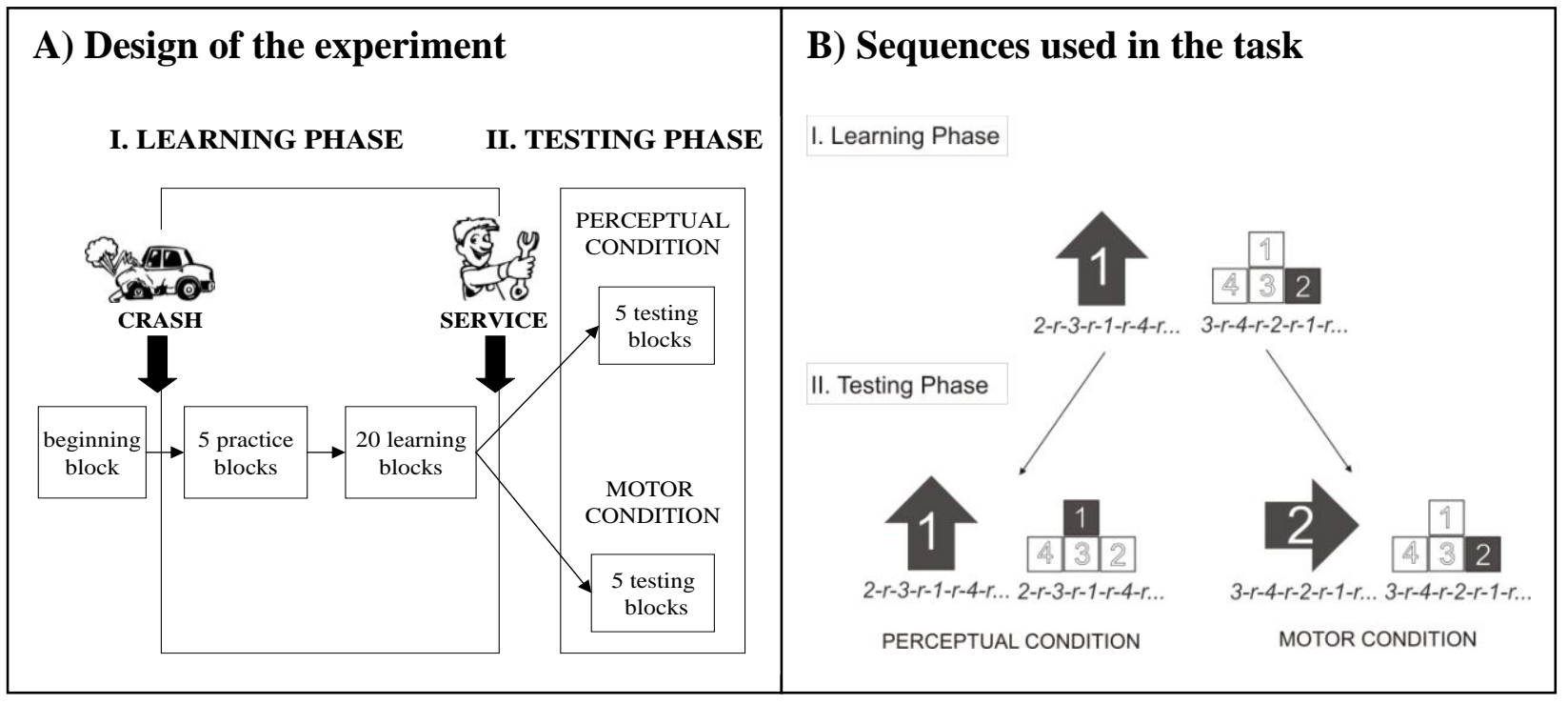

Figure 1: A) Schematic diagram of the experiment. B) In the Perceptual condition, the perceptual sequence was the same and the motor sequence (button presses) changed compared to the sequences in the learning phase. In the Motor condition, key presses followed the learned sequence and the perceptual information changed.

In the learning phase, 5 practice blocks were presented (these were excluded from the analysis), followed by 20 learning blocks with 85 key presses in each block. 
These 85 key presses included an initial 5 random presses (warm-up; excluded from the analysis), then an eight-element sequence alternated 10 times (2-R-3-R-1-R-4-R, where $\mathrm{R}$ represents random trials). The stimulus remained on the screen until the subject pressed the correct button. The next stimulus appeared after a $120 \mathrm{~ms}$ delay (response to stimulus interval, RSI) after the subject's correct response (following the parameters of the original task by J. H. Howard Jr. \& Howard, 1997). During this delay, a fixation cross was displayed on the screen. Subjects were told to respond as fast and as accurately as they could.

After the learning phase (and a 3 minute long break), the subjects were told that the car had been taken to a service station and the steering wheel had been fixed. They were told to use the answer keys corresponding to the arrows that appeared on the screen (up button for up arrow, left button for left arrow, etc.). In the testing phase, half of the subjects were assigned to the Perceptual condition and the other half to the Motor condition (see Figure 1A). In the Perceptual condition, subjects responded to the sequence seen during the learning phase (e.g., 2-R-3-R-1-R-4-R, see Figure 1B), and the appropriate key presses represented a new sequence (also $2-\mathrm{R}-3-\mathrm{R}-1-\mathrm{R}-4-\mathrm{R}$ ), which they had not practiced before. In contrast, subjects in the Motor condition had to respond by key presses practiced before (for example $3-\mathrm{R}-4-\mathrm{R}-2-\mathrm{R}-1-\mathrm{R}$, see Figure 2) but the corresponding stimuli on the screen followed another sequence (also 3-R-4$\mathrm{R}-2-\mathrm{R}-1-\mathrm{R})$, which they had not seen before. Thus, in the Perceptual condition, the perceptual sequence was the same but the motor sequence (key presses) changed compared to the previously practiced sequence. However, in the Motor condition, key presses followed the previously learned sequence and the perceptual information (the sequence of the stimuli displayed on the screen) changed. By comparing the subject's 
performance between the two conditions, we could determine whether the perceptual and the motor component had the same or different effects on learning. The possible oculomotor aspect of learning was excluded by displaying all the stimuli in the same place (in the center) of the screen.

To explore how much explicit knowledge the subject acquired about the task, we used a short questionnaire after the testing phase. None of the participants reported noticing the sequences in the tasks.

Statistical analysis - We followed the procedures of the original ASRT task $[16,17]$ in our analysis because the core structure of the tasks was the same. Given that there was a fixed sequence in the AS-RT-Race task (and in the ASRT task as well), which included alternating random elements (e.g., 2-R-3-R-1-R-4-R), some triplets or runs of three events occurred more frequently than others. For example, in the above illustration, triplets like 2_3,3_1, 1_4, 4_2 could occur more frequently because the third element could be derived from the sequence or could also be a random element. In contrast, triplets such as $4 \_1,4 \_4$ would occur less frequently because in this case, the third element could only be random. In other words, pattern trials were always high frequency, whereas one-fourth of random trials were high frequency by chance. Previous studies have shown that as participants practice, they come to respond more quickly to the high-frequency compared to the low-frequency triplets, thereby revealing sequence-specific learning (triplet type effect; $[9,18,19]$ ). In addition, general motor skill learning was revealed by the overall speed with which participants responded, irrespective of the triplet types. Thus, we obtained measures of both sequence-specific and general motor skill learning in the AS-RT-Race task. 
The blocks of the AS-RT-Race task were organized into groups of five to facilitate data processing. A group of five blocks was referred to as an epoch (a term given by the ASRT authors). The first epoch contained blocks 1-5, the second epoch contained blocks 6-10, etc. Our analysis focused only on reaction time data because subjects' accuracy remained very high during the entire test (the average was $97 \%$ for both conditions in both the learning and testing phases). Median reaction times (RT) were calculated for each subject and in each epoch both for the high and low frequency triplets.

\section{RESULTS}

Learning phase - The 2 (TRIPLET: high and low) x 4 (EPOCH: 1-4) Repeated Measures ANOVA with CONDITION (perceptual vs. motor) as the between-subject factor revealed sequence-specific learning (indicated by a significant main effect of the TRIPLET: $\left.\mathrm{F}(1,23)=124, \mathrm{MSE}=56.65, \mathrm{p}<0.001, \eta_{\mathrm{p}}{ }^{2}=0.63\right)$, as well as general motor skill learning (shown by the significant main effect of the EPOCH: $F(4,20)=8.85$, $\left.\mathrm{MSE}=32.53, \mathrm{p}<0.001, \eta_{\mathrm{p}}{ }^{2}=0.72\right)$, thereby suggesting that the more the subjects practiced, the faster their responses became (see Figure 2A-B). The two groups (perceptual and motor conditions) did not differ either in sequence-specific or in general motor skill learning ( $\mathrm{p}$ values $>0.31)$. 


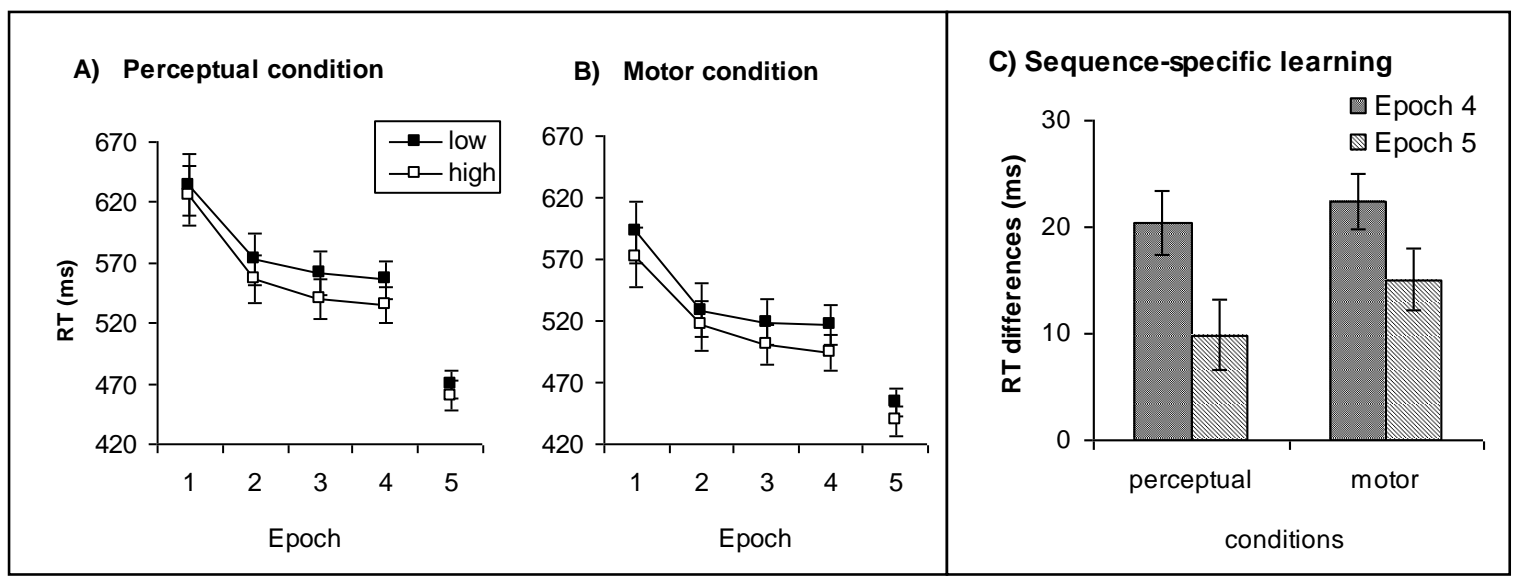

Figure 2: Results of the Learning Phase (Epoch 1-4) and Testing Phase (Epoch 5) for Perceptual (A) and Motor (B) conditions. Filled squares represent low frequency triplets; open squares represent high frequency triplets. Comparing the sequence-specific knowledge (the RT differences between high and low frequency triplets) of perceptual and motor conditions (C). Error bars indicate standard error of mean (SEM).

Testing phase - To compare the perceptual and motor conditions in the testing phase, a 2 (TRIPLET: high and low) x 2 (EPOCH: 4-5) Repeated Measures ANOVA was conducted with CONDITION (perceptual vs. motor) as the between-subject factor. The main effect of the TRIPLET was significant $(F(1,32)=69.72, M S E=139.36, p<0.001$, $\left.\eta_{\mathrm{p}}{ }^{2}=0.69\right)$ such that participants responded faster for high-frequency than for low frequency triplets (see Figure 2C). The main effect of the EPOCH was also significant $\left(F(1,32)=115.4, M S E=1448.27, p<0.001, \eta_{p}{ }^{2}=0.78\right)$, whereby subjects were faster in the testing phase (455 ms) than in the learning phase (525 ms). Interestingly, the TRIPLET $x$ EPOCH interaction was also significant $(F(1,32)=5.75, \mathrm{MSE}=117.79, \mathrm{p}=0.02$, $\left.\eta_{\mathrm{p}}{ }^{2}=0.15\right)$, thereby suggesting that the sequence-specific knowledge decreased between the learning and the testing phases (the RT difference between the high- and lowfrequency triplets was $21 \mathrm{~ms}$ in Epoch 4 and $12 \mathrm{~ms}$ in Epoch 5). However, despite this 
decrease, subjects still showed a significant triplet type effect in Epoch 5 (indicated by a one-sample t-test: $\mathrm{t}(33)=4.52, \mathrm{p}<0.001)$. In addition, there was no difference between the conditions either in sequence-specific $(\mathrm{p}=0.38)$ or in general motor skill $(\mathrm{p}=0.10)$.

\section{DISCUSSION}

Our research investigated the role of perceptual and motor learning in implicit skill learning. We addressed the possibility of demonstrating perceptual transfer beyond motor learning in a testing situation where, after the learning phase, the task continues either with motor sequence or with perceptual sequence while eliminating oculomotor learning. We were able to show learning after the learning phase both in the perceptual and motor conditions. We focused on the perceptual sequence transfer under the former condition, and the motor sequence in the latter. Our results demonstrated that under this research paradigm, both motor and perceptual transfer was significant. These results support the different methods of Song et al. [14], which demonstrated perceptual learning with probabilistic sequence learning tasks. On the other hand, our results partly differ from that of Willingham et al.[15], which did not find perceptual learning to be an important element of learning. However, their research design did not eliminate the possibility of oculomotor learning, whereas the present study did. Furthermore, our findings also indicated that there was motor transfer, thereby supporting the results of Willingham et al. [15] and their implicit motor sequence learning model.

Our findings well complement motor skill learning models [2-4], as well as the neuropsychological and neuroimaging studies that suggest the basal ganglia and the primary and secondary motor cortices play a role in implicit skill learning [2,20-22]. The task developed in the present study separated motor and perceptual learning, 
thereby allowing researchers to conduct more detailed studies in cognitive neuroscience for various pathologies affecting implicit skill learning and the underlying mechanisms of motor and perceptual learning.

\section{CONCLUSION}

In our study, we constructed a novel task (AS-RT-Race) to separate the perceptual and motor factors of implicit skill learning. We found that these components underlie the mechanisms behind skill learning to nearly the same extent. Our results draw attention to the fact that skill learning is not a single process. Instead, there are multiple mechanisms in this fundamental learning process. The novel task we developed was demonstrated to be an appropriate method to investigate the components of skill learning in different neuropsychological pathologies (e.g., basal ganglia disorders, Alzheimer's disease, etc.), and for examining the effects of development, aging and sleep on the motor and perceptual factors contributing to skill learning. 


\section{Acknowledgments}

This work was supported by the Bolyai Scholarship Program and the Hungarian National Research Fund (OTKA F 61943). We thank Ágnes Lukács, Tamás Kincses for helpful comments. 


\section{References}

[1] Reber AR. Implicit learning and tacit knowledge: An essay on the cognitive unconscious. New York: Oxford University Press; 1993.

[2] Doyon J, Bellec $\mathrm{P}$, Amsel R, Penhune V, Monchi O, Carrier $\mathrm{J}$ et al. Contributions of the basal ganglia and functionally related brain structures to motor learning. Behav Brain Res 2009; 199: 61-75.

[3] Hikosaka O, Nakahara H, Rand MK, Sakai K, Lu X, Nakamura K et al. Parallel neural networks for learning sequential procedures. TINS 1999; 22: 464-471.

[4] Hikosaka O, Nakamura K, Sakai K, Nakahara H. Central mechanisms of motor skill learning. Curr Opin Neurobiol 2002; 12: 217-222.

[5] Albouy G, Sterpenich V, Balteau E, Vandewalle G, Desseilles M, Dang-Vu T et $a l$. Both the hippocampus and striatum are involved in consolidation of motor sequence memory. Neuron 2008; 58: 261-272.

[6] Schendan H, Searl M, Melrose R, Stern C. An FMRI study of the role of the medial temporal lobe in implicit and explicit sequence learning. Neuron 2003; 37: 1013-1025.

[7] Nissen MJ, Bullemer P. Attentional requirements of learning: Evidence from performance measures. Cognitive Psychology 1987; 19: 1-32.

[8] Remillard G. Pure perceptual-based sequence learning. Journal of Experimental Psychology: Learning, Memory, and Cognition 2003; 29: 581-597.

[9] Howard JH, Jr., Howard DV. Age differences in implicit learning of higherorder dependencies in serial patterns. Psychology and Aging 1997; 12: 634-656. 
[10] Dennis NA, Howard JH, Howard DV. Implicit sequence learning without motor sequencing in young and old adults. Experimental Brain Research 2006; 175: 153-164.

[11] Robertson EM, Tormos JM, Maeda F, Pascual-Leone A. The role of the dorsolateral prefrontal cortex during sequence learning is specific for spatial information. Cerebral Cortex 2001; 11: 628-635.

[12] Deroost N, Deroost N, Coomans D, Soetens E. Perceptual load improves the expression but not learning of relevant sequence information. Experimental Psychology 2009; 56: 84.

[13] Willingham DB, Nissen MJ, Bullemer P. On the development of procedural knowledge. Journal of Experimental Psychology: Learning, Memory, and Cognition 1989; 15: 1047-1060.

[14] Song S, Howard JH, Howard DV. Perceptual sequence learning in a serial reaction time task. Experimental Brain Research 2008; 189: 145-158.

[15] Willingham DB, Wells LA, Farrell JM, Stemwedel ME. Implicit motor sequence learning is represented in response locations. Memory and Cognition 2000; 28: 366-375.

[16] Bennett IJ, Howard JH, Jr., Howard DV. Age-related differences in implicit learning of subtle third-order sequential structure. Journals of Gerontology: Series B: Psychological Sciences and Social Sciences 2007: P98.

[17] Song S, Howard JH, Jr., Howard DV. Sleep does not benefit probabilistic motor sequence learning. J Neurosci 2007; 27: 12475-12483. 
[18] Howard DV, Howard JH, Jr., Japikse K, DiYanni C, Thompson A, Somberg R. Implicit sequence learning: effects of level of structure, adult age, and extended practice. Psychol Aging 2004; 19: 79-92.

[19] Song S, Jr. JHH, Howard DV. Implicit probabilistic sequence learning is independent of explicit awareness. Learning \& Memory 2007; 14: 167-176.

[20] Grafton ST, Hazeltine E, Ivry R. Functional mapping of sequence learning in normal humans. Journal of Cognitive Neuroscience 1995; 7: 497-510.

[21] Robertson EM, Press DZ, Pascual-Leone A. Off-line learning and the primary motor cortex. J Neurosci 2005; 25: 6372-6378.

[22] Willingham DB, Koroshetz WJ. Evidence for dissociable motor skills in Huntington's disease patients. Psychobiology 1993; 21: 173-182. 